\title{
EVALUACIÓN DEL PLAN OPERATIVO INSTITUCIONAL DE LA RED DE SERVICIOS DE SALUD Y HOSPITAL RURAL TOCACHE, PERIODO: 2012-2013.
}

\section{INSTITUTIONAL ASSESSMENT OF OPERATIONAL PLAN OF THE NETWORK OF HEALTH SERVICES AND RURAL HOSPITAL TOCACHE, PERIOD: 2012-2013.}

\author{
${ }^{a}$ Dr. José Darwin Malpartida Márquez, ${ }^{\mathrm{b}} \mathrm{MSc}$. Segundo Ezequiel Ramírez Rengifo, CPC. \\ César Alberto Rengifo Romero.

\footnotetext{
${ }^{a}$ Universidad Nacional Agraria de la Selva, Perú, jose.malpartida@unas.edu.pe

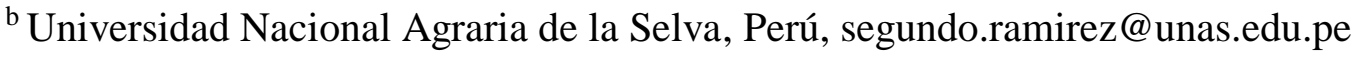 \\ ${ }^{\mathrm{c}}$ Universidad Nacional Agraria de la Selva, Perú, cesar-albett2011@ @otmail.com
}

Resumen: Tan importante como elaborar el Plan Operativo de una Institución es su evaluación, que el objetivo principal fue evaluar en función a los procedimientos técnicos en su elaboración, socialización, seguimiento y evaluación, para el logro de sus objetivos institucionales. Las encuestas se realizaron a los siguientes grupos ocupacionales, Personal administrativo (18), Personal asistencial profesional y técnico (53), estas encuestas tuvieron la finalidad de identificar el grado de participación en cada una de las fases del Plan Operativo Institucional hasta su retroalimentación, teniendo en cuenta la distribución del presupuesto por programas y las metas asignadas por programas. El trabajo de campo dio como resultado que existe una relación entre las fases que requiere el Plan Operativo Institucional y el logro de los objetivos institucionales tanto para la ejecución del presupuesto y cumplimiento de las metas en la Red de Salud y Hospital Rural de Tocache.

Palabras clave: Hospital, Plan operativo Institucional, Plan operativo por programas, Red de salud, Socialización.

Abstract: As important as developing the Operational Plan of an institution is its evaluation, That in this case objective was to evaluate according to the technical elaboration processes, 
socialization, monitoring and evaluation, to achieve their corporate goals, surveys performed the following groups ocupacionales administrative staff (18), professional and technical (53) Personal care these surveys were intended to identify the degree of participation in each of the phases of the Institutional Operational Plan to your feedback, taking into account the distribution of the program budget and program goals assigned. The field work resulted in a relationship between the phases required by the Institutional Operational Plan and the achievement of corporate goals for both budget performance and compliance goals Health Network and Tocache Rural Hospital.

Keywords: Hospital, Institutional Operational Plan, Network Health, Operating Plan Program, Socialization.

\section{INTRODUCCIÓN}

La provincia de Tocache forma parte de Región San Martin y se ubicada en la parte oriental del Perú, cuenta con dos instituciones públicas, la Red de Salud y el Hospital Regional, reciben presupuesto público y por tanto elaboran Planes Operativos Instituciones; de allí que el propósito de la investigación consistió en la evaluación de los procedimientos técnicos en su elaboración, socialización, seguimiento y evaluación para el logro de sus objetivos institucionales.

Para el logro de tales objetivos se utilizan diversas herramientas de gestión, como el "Plan Estratégico, el Plan Operativo Institucional, los Presupuestos, entre otros, pero el común denominador de todas ellas es la planificación” (Armijo, 2011, p.10), pero todas sin excepción guardan relación entre sí, se complementan unas a otras, los gestores o quienes hagan las veces necesitan contar con un mapa que visualice sus objetivos a corto y largo plazo, evaluarlas, medirlas y corregir los sesgos que se presentan en el camino. Entonces estos objetivos, desde la administración pública, son los cambios que se espera alcanzar en la realidad que se interviene, por lo general en el término de un año, y significa la solución a problemas generales identificados. Por otro lado, tenemos que el control es inherente a la actividad humana desde todos los tiempos, de manera que ha evolucionado desde la simple necesidad de seguridad individual o colectiva hasta la imperiosidad de verificar el cumplimiento de los objetivos preestablecidos y sus acciones resultantes (Plasencia, 2010). Para ello es necesario medir lo que no es medible, entonces la herramienta adecuada para este fin es el Plan Operativo Institucional (POI). Hay lugar a preguntar aspectos tales como: ¿Cuánto se ha 
avanzado al objetivo?, ¿son los recursos suficientes?, ¿se cumplen los lineamientos establecidos como políticas que integren los intereses nacionales e institucionales?, ¿el personal a cargo de todo este proceso está en condiciones de generar valor agregado a sus actividades?, preguntas que todo directivo o gestor debe hacerse al momento de asumir un cargo a fin de establecer sus metas a corto y largo plazo.

Si se continúa trabajando bajo el esquema tradicional donde las decisiones se toman sin socializar, medir y evaluar los resultados para ello el POI es una herramienta para tomar decisiones - el funcionario público trabajará en función de sus intereses o de lo que pueda hacer, pero no los de la institución, población y grupos de interés a quien debe su existencia. Además, el conocimiento profundo de la socialización en la elaboración de las herramientas de gestión institucional, propicia un proceso adecuado de integración e interacción en todos los niveles de la organización. Por esta razón, cobra importancia el diseño y planeación de difusión de la información a través de estrategias definidas que permitan la vinculación de esa parte conceptual con la parte operativa.

Pero en un mundo globalizado de hoy no se puede guiar todo desde un centralismo "cada localidad o territorio se caracteriza, por ejemplo, por una determinada estructura productiva, un mercado de trabajo, una capacidad empresarial y tecnológica, una dotación de recursos naturales e infraestructuras, un sistema social y político, una tradición y cultura, sobre los cuales se articulan los procesos de desarrollo económico" (Alburquerque, 2001, p.21), por otro lado si queremos un Estado moderno que imponga prácticas eficientes y descentralizadas (Sanin, 1999, p. 11) es necesario generar competencias en la población si queremos un gobierno de equidad aplicando el principio de Subsidiariedad (El gobierno más cercano a la población es el más idóneo para ejercer las distintas funciones que le competen al Estado). Por consiguiente, el Gobierno Nacional no debe asumir competencias que pueden ser cumplidas eficientemente por los Gobiernos Regionales y éstos, a su vez, no deben involucrarse en realizar acciones que pueden ser ejecutadas eficientemente por los gobiernos locales, evitando la duplicidad de funciones), es decir aplicando los principios que rigen la ley orgánica de Gobiernos Locales (Ley $\mathrm{N}^{\circ} 27867$, art. 8). La necesidad de generar instancias de respuesta cada vez más cercanas a los ciudadanos exige formas de 
comportamiento diferentes a las tradicionales, donde los diseños en el plano nacional deben confrontarse y articularse con los objetivos territoriales e institucionales. Esto quiere decir que se debe y obligatoriamente realizar todos los años un diagnóstico tanto de las necesidades, identificación de problemas y solución de esos problemas, ante ello elaborar un Plan Estratégico Institucional.

Ello parte de un "planeamiento para el desarrollo institucional" es decir un planeamiento aplicado a las instituciones $\mathrm{u}$ organizaciones, empresas, organizaciones sociales de base, organizaciones no gubernamentales (ONG), asociaciones, iglesias, sindicatos, etc., así como a las instituciones de naturaleza pública o privada, nacionales o extranjeras.

A diferencia del planeamiento del desarrollo nacional, regional o local, que tiene que ver con el planeamiento aplicado al desarrollo de comunidades ("planeamiento para el desarrollo comunal'), el planeamiento institucional es aplicado a instituciones que forman parte de una comunidad determinada. En otras palabras, se diferencian en el ámbito de acción, mientras que uno tiene un ámbito más global y territorial (el planeamiento para el desarrollo comunal), el otro es aplicado a las instituciones, entidades u organismos. Sin embargo, ambos deben estar debidamente articulados.

Un "Plan Estratégico Institucional" (PEI) es un instrumento orientador de la gestión o quehacer institucional de una entidad pública formulado desde una perspectiva multianual. Toma en cuenta la Visión del Desarrollo, los Objetivos Estratégicos y acciones concertadas en el Plan de Desarrollo Concertado (para el caso de las municipalidades y Gobiernos Regionales), y los lineamientos establecidos en los planes estratégicos sectoriales multianuales (Pesem). Contiene los objetivos institucionales y las acciones que le corresponde realizar en el marco de sus competencias. (Soto y Percca. 2011, p. II-1) Todo esto no debe expresar un conjunto de necesidades con un presupuesto, es necesaria su concordancia y articulación del PEI con los otros Instrumentos de Planeamiento del Sector Público, esto se refleja explícitamente en la Ley General del Sistema Nacional de Presupuesto, que textualmente señala lo siguiente:

“Las Entidades, para la elaboración de sus Planes Operativos Institucionales $y$ Presupuestos Institucionales, deben tomar en cuenta su Plan Estratégico Institucional (PEI) que debe ser concordante con el Plan 
Estratégico de Desarrollo Nacional (Plades), los Planes Estratégicos Sectoriales Multianuales (Pesem), los Planes de Desarrollo Regional Concertados (PDRC), y los Planes de Desarrollo Local Concertados (PDLC), según sea el caso" (Ley 28411, articulo 71).

Como vemos, la norma vigente hace referencia explícita que tanto los pesupuestos institucionales como los demás instrumentos del planeamiento institucional del Sector Público (POI y PEI), tienen que tener una concordancia y no es fruto solo de un conjunto de necesidades o cumplimiento presupuestal.

Desarrollado este PEI, la desagregación a nivel de actividades del presupuesto, es el Plan Operativo Institucional, el cual parte de su socialización y los mecanismos de ejecución del presupuesto priorizando el gasto, ser elaborado según las directivas presupuestales, posteriormente su monitoreo y evaluación.

\section{Metodología}

Para la determinación de la muestra se toma como referencia a todo el personal involucrado en el proceso, es decir, funcionarios, personal administrativo, profesionales de la salud tanto del hospital rural como de la zona rural. De acuerdo a los datos obtenidos por la "Red de Servicios de Salud y Hospital Rural Tocache", según la muestra calculada se realizaron 161 encuestas, las que se aplicaron en los siguientes grupos ocupacionales - entre los que se encuentran personal administrativo (42), personal asistencial profesional y técnico (119) - estas encuestas tuvieron la finalidad de identificar el grado de participación en cada una de las fases del Plan Operativo Institucional, hasta su retroalimentación, teniendo en cuenta la distribución del presupuesto por programas y las metas asignadas por programas, así mismo se analizó la ejecución porcentual del presupuesto en los años 2012 y 2013. Teniendo en cuenta la lejanía de los locales rurales se aplicó la encuesta durante los talleres y capacitaciones al personal asistencial ubicado en zonas periféricas, la encuesta se aplicó de manera indistinta hasta completar la muestra.

Por otro lado, se elaboraron tablas para verificar in situ la existencia de instrumentos de gestión y si los encargados de la toma de decisiones hacen uso de ella.

Tabla 1: Operacionalización de las variables: 


\begin{tabular}{|c|c|c|}
\hline Variables & Dimensiones & Indicadores \\
\hline $\begin{array}{l}\text { Dependiente: } \\
\text { Objetivos } \\
\text { Institucionales }\end{array}$ & $\begin{array}{c}\text { Logros } \\
\text { Institucionales }\end{array}$ & $\begin{array}{ll}\checkmark & \text { Indicadores de salud } \\
\checkmark & \text { Capacitaciones especializadas } \\
\checkmark & \text { Herramientas de gestión } \\
\checkmark & \text { Misión } \\
\checkmark & \text { Visión } \\
\checkmark & \text { Metas año 2012-2013 } \\
\end{array}$ \\
\hline \multirow{4}{*}{$\begin{array}{l}\text { Independiente: } \\
\text { Plan Operativo } \\
\text { Institucional (POI) }\end{array}$} & Socialización & $\begin{array}{ll}\checkmark & \text { Numero de capacitaciones } \\
\checkmark & \text { Programa de difusión de las } \\
& \text { medidas correctivas } \\
\checkmark & \text { Programa de difusión de los } \\
& \text { logros } \\
\checkmark & \text { Talleres previos al POI }\end{array}$ \\
\hline & Elaboración & $\begin{array}{ll} & \text { Proceso de elaboración del } \\
& \text { POI }\end{array}$ \\
\hline & Seguimiento & $\begin{array}{ll}\checkmark & \text { Talleres de capacitación } \\
\checkmark & \text { Plan de trabajo } \\
\checkmark & \text { Reuniones de trabajo }\end{array}$ \\
\hline & Evaluación & $\checkmark \quad$ Evaluación del POI \\
\hline
\end{tabular}

La presente investigación es de nivel descriptivo transversal. La confiabilidad del instrumento fue de 0.837 con la prueba de Alfa de Cronbach. Para el manejo de la información se elaboró una base de datos con el programa $\operatorname{SPSS}^{\odot}$ y se utilizó estadística descriptiva.

\section{RESULTADOS}

Con respecto a la evaluación del Plan Operativo Institucional por Programas, el trabajo de campo dio como resultado: No se cumple la fase de socialización ya que en la encuesta realizada el $50.3 \%$ refiere que no le comunicaron las metas institucionales y el $49.1 \%$ no le comunicaron los objetivos institucionales; en consecuencia, el $52.8 \%$ refiere que no está enterado de los logros y metas alcanzadas.

La población al año 2012 para la provincia de Tocache fue de 73,460 habitantes, y la población proyectada al año 2013 fue de 73,093 habitantes una disminución del $0.50 \%$, el presupuesto por programas de S/.
11’275,837 nuevos soles al año 2012 se incrementó a S/. 13’335,098 nuevos soles para el año 2013; teniendo un incremento de S/. 2’059,261 representando un crecimiento porcentual de 18.26; este incremento de presupuesto es notorio que la Red de Salud y el Hospital de Tocache no tuvieron la capacidad de lleva a cabo el 100\% del gasto, ejecutando el $4.94 \%$ para el año 2012 y el 6.7\% para el año 2013 de su presupuesto total y por programas sólo en prevención del cáncer y otros tuvieron un incremento en el gasto los demás programas tuvieron una menor inversión del gasto.

Así mismo a pesar de un incremento en el gasto público en materia de salud para la Provincia de Tocache, el impacto en las 10 primeras causas de morbilidad no es positiva, solo en las tres primeras causas hay una disminución pero en las siete restantes esta se incrementa.

Es relevante la fase de socialización, seguimiento y evaluación para que durante el proceso se puedan corregir las desviaciones y superar obstáculos, de otro lado un presupuesto desligado de la realidad social en que esta se ejecuta no conlleva a un resultado de eficiencia y eficacia en el manejo de los recursos a pesar que prácticamente se ejecutó el $100 \%$ del presupuesto los resultados en la prevención 
son adversos. En este orden de ideas la institución al no contar con un clara visión y misión, de un plan estratégico carece de identidad propia y se convierte en un mero ejecutor de presupuesto del gobierno central, por lo tanto se cumple una desconcentración de responsabilidades pero no los fines de la descentralización por lo tanto el principio de subsidiaridad queda relegado a un segundo plano.

\section{CONCLUSIONES}

La institución no cuenta con un Plan estratégico Institucional que le permita identificar los problemas institucionales y los de la localidad en materia de salud a fin de plantearse metas a corto y largo plazo.

Los programas de salud existentes entre el 2012 y 2013 siete en total obedecen a políticas en materia de salud del gobierno central y cada uno de ellos cuenta con su plan de operativo (metas y presupuesto) cuenta con un coordinador por programa y cada uno de ellos se evalúa de manera independiente.

Cada una de las fases del plan operativo, es decir socialización, elaboración, seguimiento y evaluación es realizado por programa, no se participa institucionalmente.
El proceso anual de asignación del presupuesto y metas se discuten y se evalúa a nivel de la dirección regional de salud de la Región San Martín, con los coordinadores de los programas, esto genera problemas institucionales si durante el transcurso del año uno de los coordinadores renuncia o se traslada a otra área dentro de la institución o cambio de ubicación geográfica.

\section{BIBLIOGRAFÍA}

Alburquerque, Francisco; Cortés, Patricia. (Compiladores). 2001. Desarrollo económico local y descentralización en América Latina: Análisis comparativo. Proyecto Regional de Desarrollo Económico Local y Descentralización CEPAL/GTZ. Santiago de Chile. Recuperado el 02 de abril de 2015, de http://repositorio.cepal.org/bitstream/ handle/11362/2691/S2001704_es.pdf ?sequence $=1$

Armijo, Marianela. (2011). Planificación estratégica e indicadores de desempeño en el sector público. CEPAL. Serie manuales $N^{\circ} 69$. Instituto Latinoamericano y del Caribe de Planificación Económica y Social (ILPES).

Ballart, Xavier (1992), “¿Cómo evaluar programas y servicios públicos?: Aproximación sistemática y estudio de caso". Ministerio para las Administraciones Públicas. Madrid. 
CEPLAN. (2014). Marco Conceptual del Planeamiento Estratégico. Recuperado el 20 de noviembre de 2015 , de Www.ceplan.gob.pe/documentos/m arco-conceptual-del-planeamientoestratégico: www.ceplan.gob.pe

Chiavenato, I. (2006). Introducción a la Teoría General de la Administración, 7ma. edición. México, Mc Graw Hill.

Fernández Fernández, J. M., \& Morala Gómez, B. (2006). Análisis de entidades públicas mediante indicadores de gestión: instrumento de rendición de cuentas. Pecunia: revista de la Facultad de Ciencias Económicas y Empresariales. $N^{\circ}$. 2, 79-100.

Guerrero Orozco, O. 1985. Las ciencias camerales. Revista ensayos, UNAM, México. vol. II, número 6, pp. 16-20; recuperado el 10 de novimebre de 2014 de http://omarguerrero.org/articulos/Ca meral.pdf

Guerrero Orozco, O. (1997). Principios de Administración Pública. Santa Fe de Bogotá: Escuela Superior de Administración Pública .

Kaplan, R. \& Norton P. (2004). Measuring the strategic readiness of intangible assets. Harvard Business Review.

Martínez O. Luisa María. (2013). La Planificación en el Siglo XXI. Desafíos Institucionales y Claves para un Nuevo Enfoque. Documento aprobado por la XV Conferencia Iberoamericana de Ministras y Ministros de Administración Pública y Reforma del Estado. Recuperado el 02 de marzo de http://old.clad.org/documentos/otro $\underline{\mathrm{s}-}$ documentos/La\%20Planificacion\% 20del\%20 Siglo\% 20XXI.pdf

Oviedo, Heidi Celina, \& Campo-Arias, Adalberto. (2005). Aproximación al uso del coeficiente alfa de Cronbach. Revista Colombiana de Psiquiatría, 34(4), 572-580. Retrieved March 05, 2015, from http://www.scielo.org.co/scielo.php ?script=sci_arttext\&pid=S0034$74502005000400009 \& \operatorname{lng}=$ en\&tlng $=$ es.

Plasencia Asorey, Carolina. (2010). El Sistema de Control Interno: garantía del logro de los objetivos, recuperado el 10 de noviembre de http://bvs.sld.cu/revistas/san/vol_14 5_10/san01510.htm.

Programa Pro Descentralización PRODES - USAID. (2010). Módulo Planeamiento Concertado e Institucional. Guía específica Plan operativo institucional Segunda edición actualizada. Prodes, USAID.

Ramió, C., \& Ballart, X. (1993). Lectura de Teoría de la Organización Vol. I . Madrid: Ministerio para las Administraciones Públicas. 
Dirección General de Servicios. INAP y Ministerio de la Presidencia. Boletín Oficial del Estado .

Ramió, C., \& Ballart, X. (1993). Lectura de Teoría de la Organización Vol. II . Madrid: Ministerio para las Administraciones Públicas. Dirección General de Servicios. INAP y Ministerio de la Presidencia. Boletín Oficial del Estado .

Sanín A. Hector. (1999). Control de Gestión y evaluación de resultados en la gerencia pública. Instituto Latinoamericano y del Caribe de Planificación Económica y Social ILPES. Dirección de Proyectos y Programación de Inversiones. Santiago de Chile.

Soto Cañedo, Carlos A.; Percca Rondón, María N. 2011. Proceso de Elaboración de un Plan Estratégico Institucional - PEI - Parte I. Actualidad Gubernamental N ${ }^{\circ} 30$ Abril 2011.

Stubbs, E. A. (22 de agosto de 2014). Indicadores de desempeño: naturaleza, utilidad y construcción. Recuperado el 18 de agosto 2015 de http://www.scielo.br/pdf/ci/v33n1 /v33n1a18.pdf: http://www.scielo.br

Vargas Gonzáles, V., \& Hernández Barrios, E. (2007). Indicadores de Gestión Hospitalaria. Revista de Ciencias Sociales, Vol. 13, $N^{\circ} 3,444-454$.
Normas legales y documentos de trabajo

Resolución Jefatural $\quad \mathrm{N}^{\circ}$ 003-94INAP/DNR, aprueba la Directiva $\mathrm{N}^{\circ}$ 002-94-INAP/DNR "Normas para la orientación, formulación, aplicación, evaluación y actualización del Plan de Trabajo Institucional (POI).

Ley $N^{\circ}$ 27658. Marco de la Modernización de la Gestión del Estado.

Ley $\mathrm{N}^{\circ}$ 27783. Bases de la Descentralización.

Ley $\mathrm{N}^{\circ}$ 28112. Ley marco de la Administración Financiera del Sector Público.

Ley $\mathrm{N}^{\circ}$ 28411. Ley General del Sistema Nacional de Presupuesto.

Política Nacional de Modernización de la Gestión Pública al 2021. (Documento promovido por la presidencia del Consejo de Ministros p.5-8).

Decreto supremo $\quad \mathrm{N}^{\circ}$ 054-2011-PCM. Aprueba el Plan Estratégico de Desarrollo Nacional denominado Plan Bicentenario del Perú hacia el 2021. 